() 2020 IEEE. Personal use of this material is permitted. Permission from IEEE must be obtained for all other uses, in any current or future media, including reprinting/republishing this material for advertising or promotional purposes, creating new collective works, for resale or redistribution to servers or lists, or reuse of any copyrighted component of this work in other work.

\title{
Identification of critical parameters for the design of energy management algorithms for Li-ion batteries operating in PV power plants
}

\author{
Alberto Berrueta, Adrian Soto, Javier Marcos, Íñigo de la Parra, Pablo Sanchis, Alfredo Ursúa \\ Department of Electrical, Electronic and Communications Engineering \\ Institute of Smart Cities, Public University of Navarre \\ Pamplona, Spain \\ alberto.berrueta@unavarra.es
}

\begin{abstract}
Lithium-ion batteries are gaining importance for a variety of applications due to their price decrease and characteristics improvement. For a proper use of such storage systems, an energy management algorithm (EMA) is required. A number of EMAs, with various characteristics have been published recently, given the diverse nature of battery problems. The EMA of deterministic battery problems is usually based on an optimization algorithm. The selection of such algorithm depends on a few problem characteristics, that need to be identified and closely analyzed. The aim of this paper is to identify the critical optimization problem parameters that determine the most suitable EMA for a Li-ion battery. With this purpose, the starting point is a detailed model of a Li-ion battery. Three EMAs based on the algorithms used to face deterministic problems, namely dynamic, linear and quadratic programming, are designed to optimize the energy dispatch of such battery. Using real irradiation and power price data, the results of these EMAs are compared for various case studies. Given that none of the EMAs achieves the best results for all analyzed cases, the problem parameters that determine the most suitable algorithm are identified to be four: (i) desired computation intensity, (ii) characteristics of the battery aging model, (iii) battery energy and power capabilities and (iv) number of optimization variables, which are determined by the number of energy storage systems, the length of the optimization problem and the desired time step.
\end{abstract}

Keywords-Lithium-ion battery, energy management, renewable energy, microgrid, photovoltaic plant

\section{INTRODUCTION}

Due to environmental and economical issues, renewable power plants are thriving around the World. Significant efforts are dedicated to increase the renewable power share in the electricity grid. Due to the unstable nature of such energy sources, energy storage systems (ESSs) are required for the proper operation of the electricity system. Energy management is a key issue that concerns important aspects of ESSs, such as their sizing, management, degradation and profitability. Among the available ESSs, lithium-ion batteries are an at-

We would like to acknowledge the support of the European Union under the H2020 project STARDUST (774094), the Spanish State Research Agency (AEI) and FEDER-UE under grants DPI2016-80641-R, DPI2016-80642-R, PID2019-111262RB-I00 and PID2019-110956RB-I00, the Government of Navarra through research project 0011-1411-2018-000029 GERA and the Public University of Navarre under project ReBMS PJUPNA1904. tractive option, given their outstanding power capability, high energy density and decreasing price [1].

Besides the sizing of the required Li-ion battery for an application [2], its energy management strategy has a direct impact on the system profitability, given that this is the algorithm that governs the energy dispatch to the electricity grid. On the one hand, a dispatch during the time with highest electricity price leads to higher economic income. On the other hand, the aging phenomena of the battery depend on its power flow [3]. Therefore, the management strategy should be calculated in order to enlarge the battery lifetime. Given the increasing number of applications in which a management strategy is required, this is currently a hot research topic. The design criteria of an energy management algorithm (EMA) should depend on the particular installation that needs to be governed. These criteria should be selected, based on the most relevant optimization problem parameters, with the aim of getting a trade-off between parameters such as the computation power, the robustness of the result or the required accuracy.

A number of new energy management systems (EMSs) with management algorithms adapted to different applications are being published [4]. The wide variety of energy systems using some kind of energy storage justify the various families of algorithms employed. The problems that are considered to be deterministic are tackled by one of the following approaches, which determine the complexity of the system model that can be handled. Non-linear optimizers, such as the dynamic programming [5], are used in combination with accurate, non-linear models. Other authors design models with lower complexity, being able to optimize the problem using lower computational power, with algorithms such as linear [6] or quadratic [7] programming. The inaccurate prediction of battery performance achieved by linear or quadratic models is an important concern that needs to be addressed before the design of an EMA.

On the other hand, diverse EMAs are proposed for nondeterministic problems, such as heuristic [8] and metaheuristic algorithms. Among the metaheuristic algorithms, the most remarkable are the swarm algorithms, either based on particle swarm [9], [10], ant colony [11], or ant-bee colony [12]; the genetic algorithms [13] or the taboo search algorithms 
[14]. Besides these metaheuristic optimization, other authors propose energy management systems based on fuzzy logic [15], model predictive control [16], game theory [17], or even multi-agent systems [18]. Several parameters need to be accounted for the design of a nondeterministic EMA. Among them, the performance of deterministic algorithms using different battery models provide relevant information for the design of a nondeterministic algorithm.

Previous research works have studied the performance of different deterministic EMAs in problems dealing with PV power generation [19]. The authors conclude that there is no single EMA which overcomes the others in all situations, and points out the relevance of identifying the optimization problem parameters that need to be analyzed for the proper design of a deterministic EMA. The aim of this paper is to make a close study of the typical situation of an energy storage system installed in a renewable power plant in order to identify the problem parameters that determine the most suitable deterministic EMA for the studied case. With this purpose, the most used algorithms are applied to a typical renewable plant. These EMAs are based on dynamic, linear and quadratic programming. Subsequently, their performance is analyzed and compared in order to identify the determining plant parameters and design criteria that lead to the most suitable EMA.

The remaining sections of this paper are organized as follows. Section II specifies the renewable energy plant used for the demonstration of the selected EMAs. The insights of the three studied algorithms are detailed in Section III. The first of them is based on dynamic programming, a powerful tool that deals with non-linear problems, but with high computational requirements. The second algorithm is based on linear programming, which requires a simple, linear model and a noticeably lower computational power. The third one is a quadratic-programming algorithm, which tries to find the trade-off between accuracy and simplicity. The comparison between them is detailed in Section IV, in which the three algorithms are used to compute the management strategy for the ESS in ten problems with various battery sizes, the achieved revenue is calculated for each of them and the results are contrasted. Section V details the analysis of critical problem parameters for the EMA design and, finally, Section VI summarizes the main conclusions of the paper.

\section{THE ENERGY MANAGEMENT PROBLEM: A PV-BATTERY PLANT WITH PEAK SHAVING LIMITATION}

A realistic case study is proposed in this paper in order to accomplish the comparison between the EMAs. It consists of a medium-sized PV plant with a peak power of $100 \mathrm{~kW}$ located in an industrial estate of Navarra, in the north of Spain. The following subsections describe and analyze the main data of the PV system and the Li-ion battery.

\section{A. PV system}

As shown in Figure 1, the PV system comprises the PV array and the PV inverter. The PV array peak power is $P_{\text {peak }}=$ $117.5 \mathrm{kWp}$. It is a common design strategy to slightly oversize

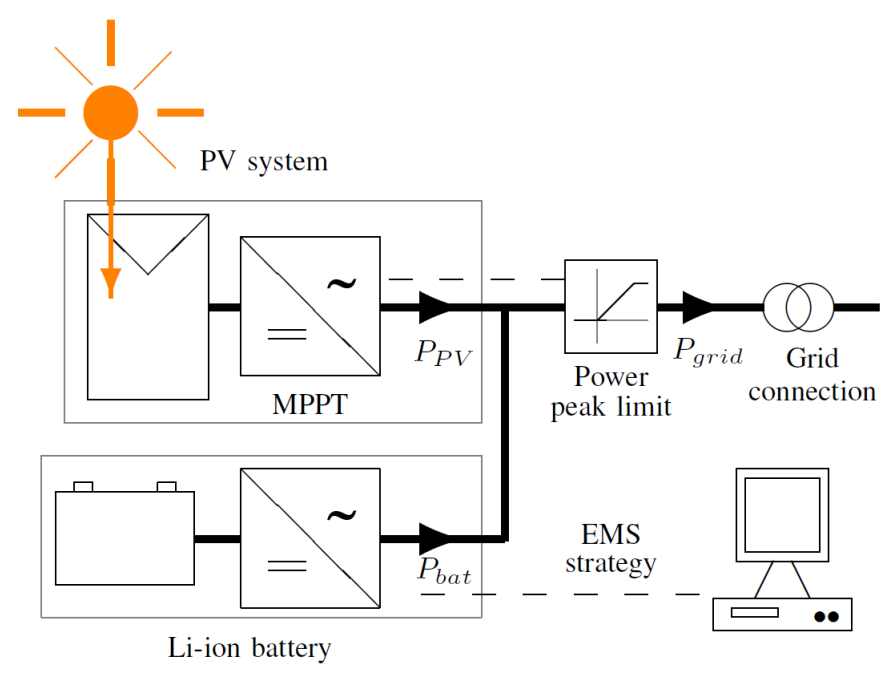

Fig. 1. System diagram of the PV+ plant.

Table I

MAIN TECHNICAL SPECIFICATIONS OF THE CASE STUDY.

\begin{tabular}{ll}
\hline Characteristic & Value \\
\hline PV panel & Yingli Solar YL250P-29b \\
PV peak power & $117.5 \mathrm{~kW}$ \\
PV inverter & Ingeteam IS 3PLAY \\
PV inverter rated power & $100 \mathrm{~kW}$ \\
Battery converter & FeCon BAT50 \\
Battery converter power & $50 \mathrm{~kW}$ \\
Feed in limitation & $60 \%$ of the inverter power \\
Grid voltage & $13.2 \mathrm{kV}^{-2}$ \\
Annual solar radiation & $1,700 \mathrm{kWh} \mathrm{m}^{-2}$ \\
\hline
\end{tabular}

the PV field compared to the inverter. In this case, $P_{P V, i n v}=$ $0.85 \cdot P_{\text {peak }} \approx 100 \mathrm{~kW}$.

The control algorithm of the PV inverter is the maximum power point tracker (MPPT). In a normal situation, the MPPT achieves maximum $\mathrm{PV}$ power $\left(P_{P V}=P_{P V}^{*}\right)$. However, as the saturation of the distribution network is a concern in industrial estates [5], [20], a feed-in power limitation is considered. The selected limiting value is $60 \%$ of the inverter nominal power, as set by the German Federal Government in the market incentive program for PV Battery Systems PV-Speicher [21]. Therefore, when a power higher than the maximum feed-in power $\left(P_{\text {grid,max }}\right)$ can be injected into the grid, the inverter power is limited, as shown in Figure 1 and the extra available power is not generated $\left(P_{P V} \leq P_{P V}^{*}\right)$. Measured meteorological data in Spain are taken from the freeaccess database Meteonavarra [22]. The mentioned technical characteristics of the case study are summarized in Table I.

\section{B. Li-ion battery}

In order to model the battery electrical performance, the equivalent circuit shown in Figure 2 (a) is used [23]. It consists of a voltage source dependent on the state of charge $v_{O C}(S O C)$ [24], as represented in Figure 2 (b), and an internal series resistance $R_{i}$ which also includes the dependency on battery SOC, see Figure 2 (c). This model predicts the battery efficiency with high accuracy, given that it takes into account 
(a)

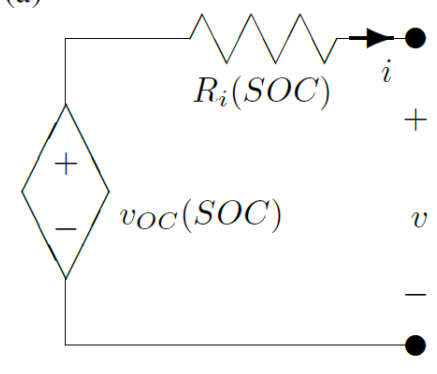

(b)

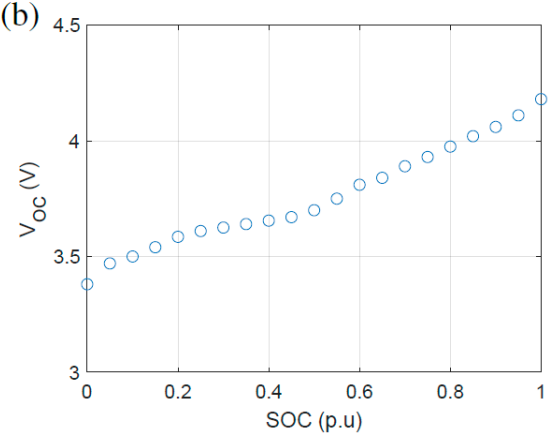

(c)

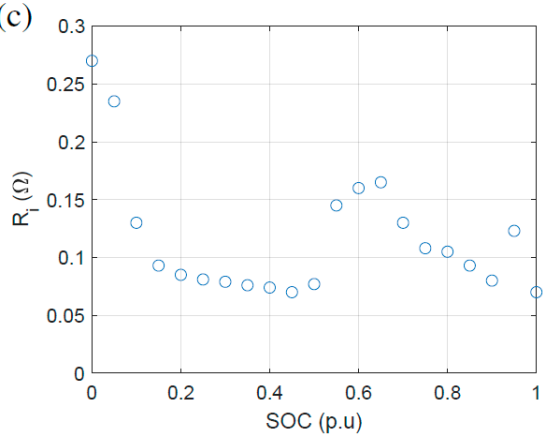

Fig. 2. Cell electrical model: Equivalent circuit (a), $v_{O C}-S O C$ relationship (b) and $R_{i}-S O C$ relationship (c) [5].

its current and SOC dependencies. The SOC defines the stored capacity relative to the actual full capacity $(C(S O H))$ :

$$
S O C(t)=S O C\left(t_{0}\right)-\int_{t_{0}}^{t} \frac{i_{b a t}(t)}{C(S O H)} d t
$$

The battery aging has a capital importance for the energy management strategy, given that the aging phenomena are determined by the battery use. As commonly done with Liion batteries, its aging effects are divided into calendar and cycle aging, which are assumed to be independent of each other [25]. A linear time dependency of capacity fade and resistance rise is assumed for the calendar aging, as shown in Equation 2 and Equation 3. For the cycle aging modeling, the dependency of capacity fade and impedance rise with the number of equivalent cycles $Q$ is assumed to be also linear:

$$
\begin{gathered}
\frac{\Delta C(t, Q)}{C(t)}=-\left(\alpha_{C} \cdot t+\beta_{C} \cdot Q\right) \\
\frac{\Delta R_{i}(t, Q)}{R_{i}(t)}=\alpha_{R_{i}} \cdot t+\beta_{R_{i}} \cdot Q
\end{gathered}
$$

where $t$ is expressed in years and $Q$ in equivalent full cycles.

Parameters $\alpha_{j}$ ( $j$ representing $C$ and $R_{i}$ ) determine the calendar aging, while $\beta_{j}$ govern the cycle aging. The dependencies of these four parameters are expressed, as proposed in [5], by the following equations:

$$
\begin{aligned}
\alpha_{j} & =a_{v, j} \cdot\left(v_{c e l l}-a_{0, j}\right) \cdot \exp \left(-\frac{a_{T, j}}{T}\right) \\
\beta_{i} & =b_{0, j}+b_{v, j} \cdot\left(v_{c y c}-b_{v 0, j}\right) .^{2}+b_{D O D, j} \cdot D O D \\
& +b_{I, j} \cdot \exp \left(b_{\exp } \cdot \frac{\left|i_{b a t}\right|}{C}\right)
\end{aligned}
$$

Considering that the battery lifetime is over when its capacity fade or impedance rise reach $20 \%$, of the rated values, $\triangle S O H$ is defined as follows:

$$
\Delta S O H=-\frac{1}{0.2} \cdot \max \left[\left|\frac{\Delta C}{C}\right|,\left|\frac{\Delta R_{i}}{R_{i}}\right|\right]
$$

\section{Economical scenario and objective function}

The energy management strategies are designed to maximize the revenue of the PV-battery system. Therefore, the objective function $J$ is an economic revenue obtained by the inclusion of a battery in the PV plant. This revenue comes from the combined effect of the augmented PV energy injected into the grid and the displacement of the PV generation to the time interval with highest energy price. The battery aging costs are accounted by taking battery aging into account. This function takes shape into the following equation:

$$
\begin{gathered}
J=\int_{t_{0}}^{t}\left[\left(P_{\text {grid }, \mathrm{PV}-\text { bat }}-P_{\text {grid }, \mathrm{PV}}\right) \cdot P C_{\text {elec }}+\right. \\
\left.\Delta S O H \cdot P C_{\text {bat }}\right] d t
\end{gathered}
$$

The economic variables involved in this expression are $P C_{\text {elec }}$, which is the price of electricity (in EUR per $\mathrm{kWh}$ ), and $P C_{b a t}$, which is the purchase price of the Li-ion battery (in EUR).

It is interesting to make the analysis under realistic, variable electricity prices. However, some grid services are not properly remunerated, based on current market rules. Therefore, the real electricity price in Spain is scaled up to an average price of EUR 0.14 per kWh maintaining the current variability of market prices. A price of EUR 250 per $\mathrm{kWh}$ for the battery system is considered, which includes the cost of the battery power converter.

\section{ENERGY MANAGEMENT STRATEGIES FOR LI-ION BATTERIES}

The three deterministic algorithms used by energy management systems (EMSs) are detailed in this section. Besides their features, the required adaptations of the management problem, such as simplification or linearization of some expressions, are compiled in the subsequent subsections.

\section{A. Dynamic programming}

The dynamic programming tackles a complex, sequential optimization problem by breaking it down into several simpler sub-problems. The main problem is solved thanks to the Bellman Principle of Optimality, which states that a decision subsequence of the optimal decision sequence is also an optimal solution of the corresponding subproblem among the subsequences with the same final state [26].

The dynamic programming is a suitable algorithm for EMSs, given the sequential and non-linear nature of these problems. However, its main drawbacks are the high computational requirements and the exponential increase of the problem complexity entailed by an increasing number of variables. In a problem such as the one studied in this paper, 
with a temporal frame imposed by the solar radiation (one day), the number of variables limit the minimum time step that can be tackled. As a trade-off between reasonable computing time and results accuracy, a time step of one hour is chosen.

The algorithm uses the model and objective function detailed above, along with the following operational constraints:

$$
\begin{aligned}
v_{\text {bat }, \text { min }} \leq v_{\text {bat }} & \leq v_{\text {bat } \text { max }} \\
S O C_{\text {min }} \leq S O C & \leq S O C_{\text {max }} \\
i_{\text {bat }, \text { min }} \leq i_{\text {bat }} & \leq i_{\text {bat }, \text { max }} \\
P_{\text {grid }, \text { min }} \leq P_{\text {grid }} & \leq P_{g r i d}^{*} \\
P_{P V} & \leq P_{P V}^{*} \\
P_{b a t} & \leq P_{N, \text { conv }}
\end{aligned}
$$

\section{B. Linear programming}

A lineal algorithm finds the optimal value of a linear function, subject to linear equality and inequality constraints. Its main advantage is the reduced computational requirement. However, the linear fits required by this technique do not allow the use of non-linear models or constraints. This issue entails concerns about the loss of accuracy of linear algorithms.

Specifically, three expressions of the model explained above need to be modified in order to use a linear algorithm:

- The relationship $v_{O C}(S O C)$ cannot be represented by measured data stored in a lookup table. By contrast, a linear expression needs to be fitted. Figure 3 (a) shows this fit, which provides a value of $R^{2}=0.977$.

- The efficiency of the battery cannot be modeled by the internal resistance, $R_{i}$, since a resistance implies power losses proportional to the square of the current: $P_{\text {loss }}=$ $R_{i} \cdot I_{b a t}^{2}$. For a linear algorithm to be applied, the power losses need to be considered as a constant value.

- The cycle aging term $\beta_{i}$ (see Equation 5) has an exponential term, which needs to be linearized. As represented in Figure 3 (b), a linear expression cannot properly fit this exponential trend of the cycle aging for a wide current range. Therefore, the linearization needs to be performed by taking the battery current into account. Three linear fits are shown in this figure, considering battery current rates below $0.5 \mathrm{C}$, below $1 \mathrm{C}$ and below $1.5 \mathrm{C}$. Given that the time step adopted in this paper is $1 \mathrm{~h}$, the maximum battery current that can be calculated is $1 \mathrm{C}$. Therefore, this is the chosen fit, which provides a value of $R^{2}=0.90$.

\section{Quadratic programming}

A quadratic optimization algorithm deals with quadratic objective functions, even though the constraints need to be linear. Given that the constraints of the studied problem are already linear equations, this algorithm requires lower simplifications of the model than a linear algorithm. Specifically, an internal resistance $R_{i}$ can be modeled by means of a quadratic model, even though its dependency with SOC cannot be taken into account. This improves the simplification of a constant battery efficiency, including its dependency on the managed power. Another improvement of a quadratic objective function, compared to a linear one, is the inclusion of the dependency of cycle aging (parameter $\beta_{i}$ of Equation 5) on battery current. Additionally, the relationship $v_{O C}(S O C)$ shown in Figure 3 (a) can be fitted by a second-order polynomial expression, as shown in Figure 3 (c). These three improvements make the quadratic model more realistic than a linear one.

Among these three enhancements, the most relevant one is the aging modeling, which can be seen by comparing Figure 3 (b) and (c). The coefficient of determination of these two fits for currents lower than $1 \mathrm{C}$ are $R_{\text {linear }}^{2}=0.90$ and $R_{\text {quadratic }}^{2}=0.99$.

\section{PERformance COMPARISON OF ENERGy MANAGEMENT STRATEGIES}

A comparison between the algorithms explained above, used to calculate the energy management of a Li-ion battery, is presented in this section. Special attention is devoted to:

- Computation requirements.

- Applied battery model (the most accurate available, its linear fit, or the quadratic fit).

- Computed management strategy and achieved revenue.

- Problem characteristics such as time step, battery size and maximum battery power.

A flow diagram of this comparison is shown in Figure 4. The input data for the optimization problem are the renewable power generated by the plant during a whole year, the hourly price offered in the electricity market and the battery size. The three algorithms explained in the previous section, along with the appropriate model, are used to calculate three different management strategies for the year. As explained above, the most accurate model available for the system has a number of non-linear relationships. Therefore, it needs to be simplified in order to be used by the quadratic and linear algorithms. The economic income and aging of the battery after the year of operation following the three management strategies are calculated by means of the non-linear, accurate model. Finally, the revenue obtained by each algorithm is computed by the subtraction of the battery aging cost from the economic input achieved by selling electricity. For better comparison, the revenue is provided as relative values, normalized with the revenue obtained by the dynamic algorithm as follows:

$$
\text { Relative revenue }=\frac{\text { Studied revenue }}{\text { Dynamic algorithm revenue }}
$$

Given that the ratio between the battery and the plant size determine the variable discretization required for the dynamic algorithm, ten problems with battery sizes ranging from $0.10 \mathrm{kWh} / \mathrm{kWp}$ to $2.6 \mathrm{kWh} / \mathrm{kWp}$ are solved using each algorithm. The battery size is defined as follows:

$$
\mathrm{kWh} / \mathrm{kWp}=\frac{\text { Battery capacity }(\mathrm{kWh})}{\mathrm{PV} \text { peak power }(\mathrm{kWp})}
$$

For the sake of comparability, the optimization problem in all the cases is reduced to one day, which requires the imposition of a battery SOC at the beginning and at the end of each day. Then, 365 problems are solved in order to compute the management strategy for the whole year. 

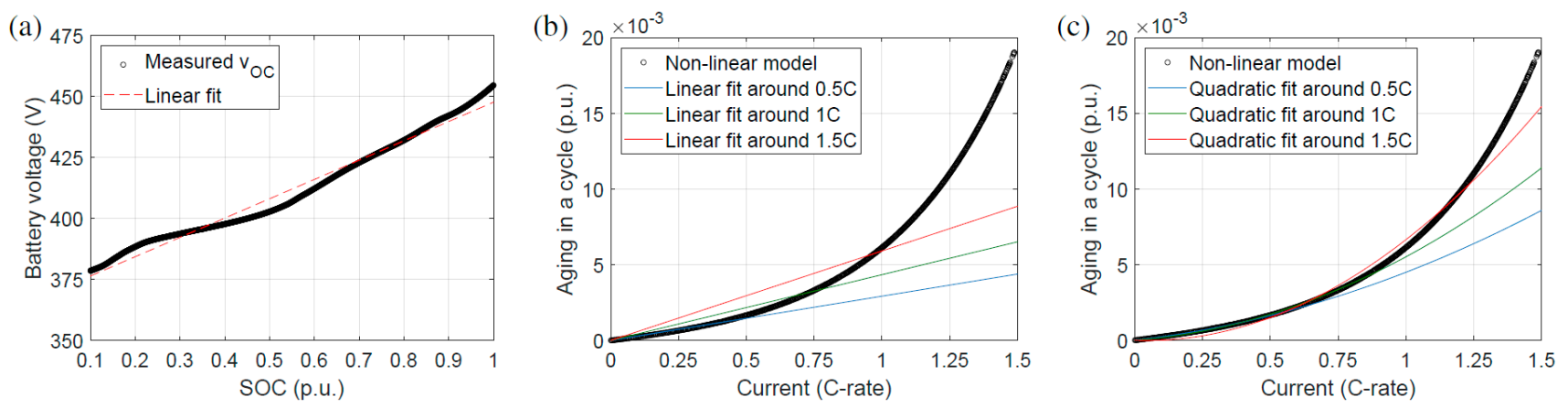

Fig. 3. Comparison between the non-linear model and the fittings of the linear and quadratic models: (a) linear fit of $v_{O C}(S O C)$, (b) linear fit of cycle aging ( $\beta_{i}$ in Equation 5), and (c) quadratic fit of cycle aging ( $\beta_{i}$ in Equation 5).

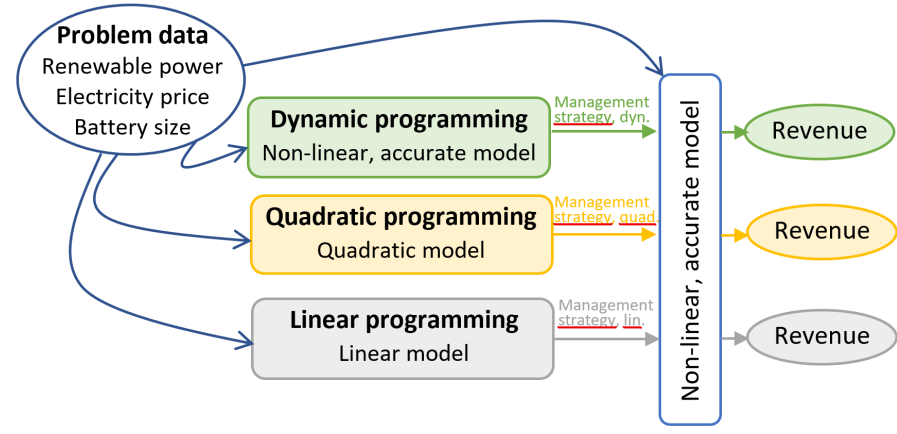

Fig. 4. Flow diagram of the comparison between the three algorithms, represented in different colors, based on uniform database and revenue calculation.

The comparison between the dynamic and the linear algorithms is shown in Figure 5. Figure 5 (a) shows the battery state of charge. Even though both algorithms calculate similar strategies, based on a battery charge using a surplus of PV power and battery discharge during the evening, there is an interesting difference. While the dynamic algorithm schedules a soft battery discharge during the whole evening, even though the electricity price has slight variations, the linear algorithm schedules a high-power battery discharge during the hour with highest electricity price. This is due to the linear fit of the aging model, explained in the previous section, that prevents the inclusion of the battery current as an input variable. However, this strategy leads to a faster battery aging, which reduces the obtained revenue. This is the reason why the relative revenue shown in Figure 5 (b) is lower than $100 \%$ for most of the battery sizes, giving an average value of $80 \%$ for the ten sizes analyzed in this paper. The linear algorithm has a lower performance for installations with small batteries, providing a revenue as low as $50 \%$ of that obtained by a dynamic program. This is due to the repetitive power peaks scheduled by the linear model. It is noteworthy that the linear algorithm achieves better results than the dynamic for large batteries. This effect is due to the combination of two issues. On the one hand, given that the power of the battery inverter is not changed, there is no fast discharge capability in the system for large batteries, thereby mitigating the main drawback of the linear algorithm, which tries to schedule a fast battery discharge during the hour with highest electricity price. On the other hand, the step size of the discrete variables required for a dynamic optimizer increases as the battery is enlarged, reducing the accuracy of the dynamic algorithm.

A summary of this comparison is provided in Table II. The second column is related to the dynamic algorithm, while the third one concerns the linear optimizer. Specially remarkable from this table is the row of computation time. The time required by a typical desktop computer to calculate the dispatch plan for a year is reduced from $2514 \mathrm{~s}$ used by the dynamic optimizer to $10.9 \mathrm{~s}$ required by the linear algorithm. This reduced complexity leads to the following row, in which the minimum time step that can be managed by each algorithm in order to solve one-day problems is provided. The shorter the time step, the larger the number of variables and, therefore, the higher the computation requirements. A linear algorithm can solve problems with a $30 \mathrm{~s}$ time step, while the dynamic algorithm is unable to solve the problem if the time step is shorter than $1 \mathrm{~h}$.

Analogously, Figure 6 shows the comparison between the dynamic and quadratic algorithms. It can be seen in Figure 6 (a) that the schedule computed by both algorithms is similar. The most notorious difference is that the quadratic algorithm schedules a slower charging process than that of the dynamic program. This leads to enhanced calendar aging, given its dependency on battery voltage, but reduced cycle aging, given its dependency on current. These two processes are similar and their overall effect is negligible, as can be seen in Figure 6 (b). The quadratic algorithm achieves a similar revenue than the dynamic optimizer for all battery sizes, being the average revenue a $102 \%$ compared to that obtained by the dynamic algorithm. Similar to the previous analysis, the relative revenue increases for large battery sizes as a result of the inaccuracy of the dynamic algorithm induced by larger discretization steps.

The fourth column of Table II summarizes the main characteristics of the quadratic algorithm. It achieves a good tradeoff between accuracy and simplicity. The computation time is reduced from $2514 \mathrm{~s}$ required by the dynamic program to only $13.2 \mathrm{~s}$ for a one-year simulation. Meanwhile, the obtained revenue is similar to that achieved by the dynamic program, being the average value even $2 \%$ higher. It is remarkable to note that the quadratic fit shown in Figure 3 is worse for higher current. Therefore, a battery current lower than $1.5 \mathrm{C}$ is required for a suitable performance of this algorithm. 
(a)

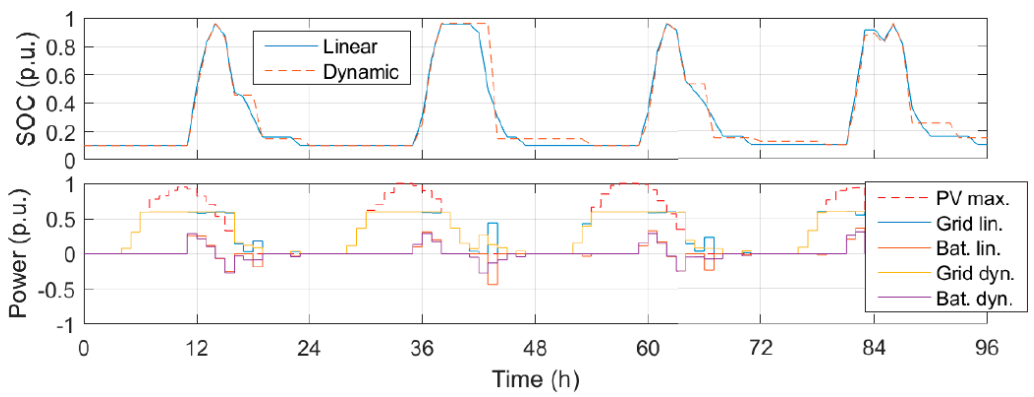

(b)

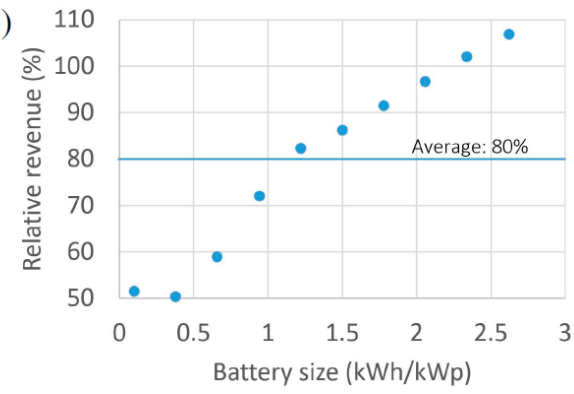

Fig. 5. Performance of the linear algorithm compared to the dynamic algorithm: (a) energy dispatch calculated for four days, with battery SOC on the top and system powers on the bottom, and (b) relative revenue between the linear and the dynamic algorithms.

(a)

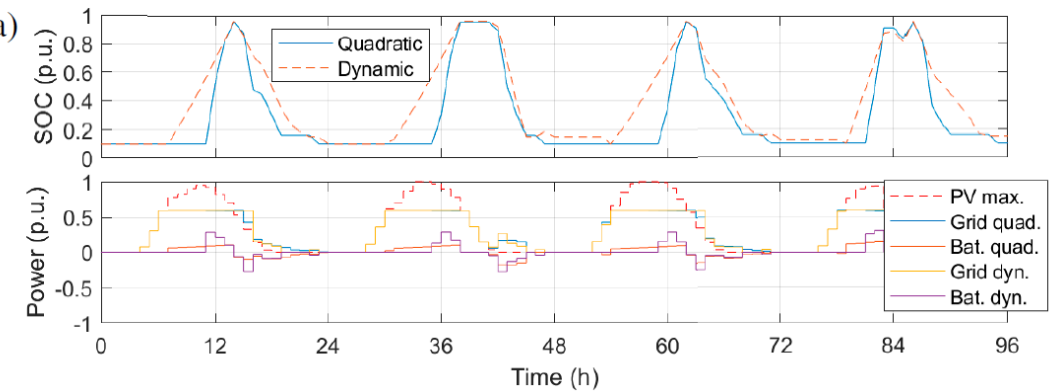

(b)

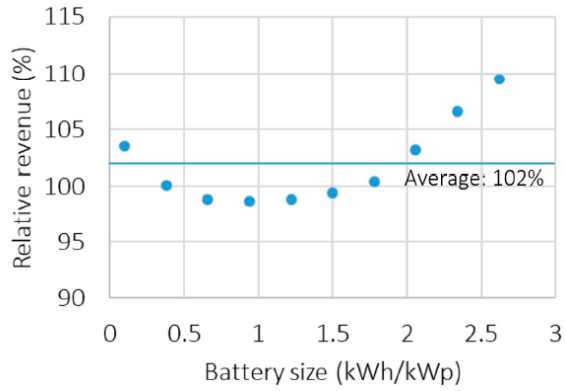

Fig. 6. Performance of the quadratic algorithm compared to the dynamic algorithm: (a) energy dispatch calculated for four days, with battery SOC on the top and system powers on the bottom, and (b) relative revenue between the quadratic and the dynamic algorithms.

Table II

MAIN CHARACTERISTICS OF THE THREE DETERMINISTIC EMAS.

\begin{tabular}{|c|c|c|c|}
\hline & Dynamic & Linear & Quadratic \\
\hline $\begin{array}{l}\text { Main sources of } \\
\text { inaccuracy }\end{array}$ & $\begin{array}{l}\text { Discretization required, } \\
\text { notorious with large } \\
\text { batteries }\end{array}$ & $\begin{array}{l}\text { Cycle aging independent of power } \\
\text { Constant efficiency } \\
\text { Linear fit of } v_{O C}(S O C)\end{array}$ & $\begin{array}{l}R_{i} \text { independent of SOC } \\
\text { Joule efficiency } \\
\text { Non-exponential cycle aging }\end{array}$ \\
\hline $\begin{array}{l}\text { Remarkable } \\
\text { characteristics }\end{array}$ & $\begin{array}{l}\text { Loss of optimality because } \\
\text { of discrete variables }\end{array}$ & $\begin{array}{l}\text { Higher current and faster aging } \\
\text { Poor performance for small battery }\end{array}$ & $\begin{array}{l}\text { Trade-off between accuracy } \\
\text { and computation requirements }\end{array}$ \\
\hline Computation time & $2514 \mathrm{~s}$ & $10.9 \mathrm{~s}$ & $13.2 \mathrm{~s}$ \\
\hline Minimum time step & $1 \mathrm{~h}$ & $30 \mathrm{~s}$ & $30 \mathrm{~s}$ \\
\hline Max. battery current & No loss of accuracy & $0.5 \mathrm{C}$ & $1.5 \mathrm{C}$ \\
\hline Average rel. revenue & $100 \%$ & $80 \%$ & $102 \%$ \\
\hline $\begin{array}{l}\text { Preferred } \\
\text { applications }\end{array}$ & $\begin{array}{l}\text { Off-line, accurate } \\
\text { battery model }\end{array}$ & $\begin{array}{l}\text { On-line, regular processor } \\
\text { Limited to low power }\end{array}$ & $\begin{array}{l}\text { On-line, low range processor } \\
\text { Off-line, uncritical accuracy }\end{array}$ \\
\hline
\end{tabular}

\section{PRoblem PARAMETERS FOR THE DESIGN OF A SUITABLE ALGORITHM}

As detailed in the previous sections, the performance of the energy management algorithm for an energy storage system varies depending on the application. In this section the most relevant parameters of such problems are compiled in order to establish the design criteria to achieve a suitable BMS. Once these criteria are established, the particular application data need to be used in order to develop the proper EMS for the application.

\section{A. Algorithm for real time or for offline simulations}

A good algorithm for offline simulations may not be suitable for real-time applications. While the aim of offline algorithms is to reach conclusions about the best sizing of the energy storage system, real-time algorithms are designed to manage the ESS during its operation.
Therefore, the computational effort is not a limiting factor for offline algorithms, which can be run in powerful computers. However, they need to accurately predict the battery aging in order for the ESS to last during the whole expected lifetime. Non-linear algorithms using accurate aging models may be suitable for this kind of applications. By contrast, realtime algorithms run in low-power micro-controllers and need to optimize the energy management in the short term. Based on the results shown in Table II, linear or quadratic algorithms can be suitable options for these applications.

\section{B. Characteristics of the available aging model}

The results provided by energy management algorithms have a high sensitivity on the battery aging model. Depending on the particular Li-ion battery used in the application and on the performance characteristics (maximum current, temperature, etc.), the designed aging model might differ. Taking the 
variables of the aging model into account, the linearization of the model should be analyzed as explained in Section III, and the associated loss of accuracy needs to be quantified.

In the event of having a highly accurate aging model for the used battery, and once every simplifications have been proven to drastically reduce its accuracy, the non-linear algorithm is the most suitable option. However, in most cases the aging models are not able to predict the battery behavior with high accuracy. In such cases, a model simplification in order to use a quadratic EMS might not reduce the usefulness of the obtained results.

\section{Number of problem variables}

The number of variables involved in the optimization problem is a critical parameter to be taken into account, given the rise in complexity entailed by a higher number of variables. Therefore, the following three aspects should be considered:

- Length of the problem: PV problems can be restricted to one day, which is not the case of systems based on wind energy.

- Required time step: in applications with fast fluctuation, a reduced time step can be critical for the suitable prediction of battery lifetime [27].

- Hybridization of energy storage systems: if the energy dispatch plan of more than one ESS needs to be computed, the number of variables increases consequently.

As detailed in previous sections, a non-linear energy management algorithm is not able to handle much more than 24 variables. Therefore, if any of the previous aspects entails an increase in the number of problem variables, a quadratic or linear algorithm are the most suitable choice.

\section{Battery size (energy and power)}

Non-linear algorithms can only deal with discrete battery SOC values, and a larger number of discretization steps entails an increase of the problem complexity. A reasonable size of such discretization steps is $\triangle S O C \approx 1 \%$. The amount of energy involved in each $\triangle S O C$ increases with the battery size. Hence, in a problem with a relatively large battery, each $\triangle S O C$ has remarkable effect in the system operation.

Therefore, for a small battery (smaller than $1 \mathrm{kWh}$ per $\mathrm{kWp}$ of PV plant in a system), a non-linear algorithm can reach a good solution by means of accurate battery models. However, if the battery is larger, the error introduced by the larger $\triangle S O C$ overshadows the higher performance of the accurate, non-linear model, thereby deteriorating the result. As a consequence, a quadratic or linear energy management algorithm is preferred for systems with larger battery energy.

The relevance of battery power is due to its effect over battery degradation. As detailed in Section III, this dependency cannot be fit by means of a linear model for C-rates larger than $0.5 \mathrm{C}$. Meanwhile, the fitting of a quadratic model provides suitable results for $\mathrm{C}$-rates up to $1.5 \mathrm{C}$ and a non-linear model can be designed to predict battery aging at any $\mathrm{C}$-rate.

\section{CONCLUSION}

This paper identifies the critical optimization problem parameters to take into account for the design of an energy management algorithm suitable for the application under study. Specifically, the algorithms that tackle deterministic problems are analyzed, namely linear, quadratic and dynamic programming. The main conclusion is that four problem parameters are critical for the selection of an EMA:

- Computation intensity

- Characteristics of the battery aging model

- Number of optimization variables

- Battery size (energy and power)

The analysis of these four parameters lead to the tentative suitability criteria shown in Table III. Even though this table is a good tool for a first selection of an EMA, each problem needs to be closely analyzed as explained in the paper.

The most notorious difference among the algorithms is the computation time. A commercial PC takes 42 minutes to compute the energy dispatch during a year by means of a dynamic algorithm, while the linear and quadratic algorithms can be run in about $10 \mathrm{~s}$. This large computation time limits the applicability of dynamic algorithms in two main aspects. Firstly, a larger number of variables entails a great increase of the problem complexity. A commercial PC is not able to solve such problem with much more than 24 variables. Therefore, each optimization problem needs to be reduced to one day, and the minimum time step that can be considered is $1 \mathrm{~h}$. This limitation is not critical for the management of PV systems, due to its daily profile, but can jeopardize the use of a dynamic algorithm for other systems, such as wind power plants. The second restriction is its limitation to discrete variables. This entails a divergence between the achieved solution and the actual optimum, which depends on the discretization step. Given that larger batteries require larger discretization steps, a dynamic algorithm achieves solutions farther from the optimum.

By contrast, the use of linear and quadratic algorithms requires a number of simplifications in the system model, objective function, and constraints that can entail a deviation in the optimization. The analysis herein presented quantifies the average loss of optimality for the linear algorithm in $20 \%$. The main reason is that the current is not taken into account as an accelerator factor for the battery aging. On the other hand, the quadratic algorithm offers a trade-off between simplicity and accuracy. The average result for the analyzed scenarios overcomes the performance of the dynamic algorithm, and its computational requirements are notably lower. However, the quadratic fit presented in Figure 3 (c) shows a fit loss for high currents. Therefore, worse results are expected for the quadratic algorithm if the maximum battery current surpasses $1.5 \mathrm{C}$. Given the low current required for the renewable-energy application analyzed in this paper, this loss of optimality is not characterized.

To sum up, this paper establishes a tool to approach the design of a proper energy management algorithm. If accuracy is a main concern and the battery has a low energy capacity compared to the power, a dynamic algorithm may be a suitable 
Table III

CRITERIA THAT DETERMINE THE MOST SUITABLE EMA.

\begin{tabular}{lccc}
\hline Requirement & Dyn. & Lin. & Quad. \\
\hline On-line execution & $x$ & $\checkmark$ & $\checkmark$ \\
Low-range processor & $x$ & $\checkmark$ & $x$ \\
Accurate aging prediction & $\checkmark$ & $x$ & $x$ \\
Problems longer than 1 day & $x$ & $\checkmark$ & $\checkmark$ \\
Time step shorter than 1 h. & $x$ & $\checkmark$ & $\checkmark$ \\
Hybrid ESS & $x$ & $\checkmark$ & $\checkmark$ \\
Battery size $>1 \mathrm{kWh} / \mathrm{kWp}$ & $x$ & $\checkmark$ & $\checkmark$ \\
Bat. power $>1.5 \mathrm{C}$ & $\checkmark$ & $x$ & $x$ \\
$0.5 \mathrm{C}<$ Bat. power $<1.5 \mathrm{C}$ & $\checkmark$ & $x$ & $\checkmark$ \\
\hline
\end{tabular}

choice. However, if the energy capacity of the battery is large compared to its maximum power, or problems with more than 24 variables need to be optimized, a quadratic algorithm may be a more clever option. Finally, linear algorithms have been proven not to be able to properly model the battery aging, and provide an average profit $20 \%$ lower than the maximum achievable, but can be the most suitable option for situations in which the computation power is required to be extremely low.

\section{REFERENCES}

[1] T. Bunsen, P. Cazzola, M. Gorner, L. Paoli, S. Scheffer, R. Schuitmaker, J. Tattini, and J. Teter, "Global EV outlook 2018: Towards cross-modal electrification," International Energy Agency, Technical report, 2018.

[2] I. San Martín, A. Berrueta, P. Sanchis, and A. Ursúa, "Methodology for sizing stand-alone hybrid systems: A case study of a traffic control system," Energy, vol. 153, pp. 870 - 881, 2018.

[3] A. Berrueta, J. Pascual, I. San Martín, P. Sanchis, and A. Ursúa, "Influence of the aging model of lithium-ion batteries on the management of PV self-consumption systems," in EEEIC / I CPS Europe, 2018, pp. $1-5$.

[4] M. S. Alam and S. A. Arefifar, "Energy management in power distribution systems: Review, classification, limitations and challenges," IEEE Access, vol. 7, pp. 92979-93 001, 2019.

[5] A. Berrueta, M. Heck, M. Jantsch, A. Ursúa, and P. Sanchis, "Combined dynamic programming and region-elimination technique algorithm for optimal sizing and management of lithium-ion batteries for photovoltaic plants," Applied Energy, vol. 228, pp. 1 - 11, 2018.

[6] F. Y. Melhem, O. Grunder, Z. Hammoudan, and N. Moubayed, "Energy management in electrical smart grid environment using robust optimization algorithm," IEEE Transactions on Industry Applications, vol. 54 no. 3, pp. 2714-2726, 2018.

[7] T. G. Paul, S. J. Hossain, S. Ghosh, P. Mandal, and S. Kamalasadan, "A quadratic programming based optimal power and battery dispatch for grid-connected microgrid," IEEE Transactions on Industry Applications, vol. 54 , no. 2, pp. $1793-1805,2018$

[8] A. Jindal, N. Kumar, and J. J. P. C. Rodrigues, "A heuristic-based smart HVAC energy management scheme for university buildings," IEEE Transactions on Industrial Informatics, vol. 14, no. 11, pp. 5074-5086, 2018.

[9] S. A. Pourmousavi, M. H. Nehrir, C. M. Colson, and C. Wang, "Realtime energy management of a stand-alone hybrid wind-microturbine energy system using particle swarm optimization," IEEE Transactions on Sustainable Energy, vol. 1, no. 3, pp. 193-201, 2010.

[10] U. Sarma and S. Ganguly, "Determination of the component sizing for the PEM fuel cell-battery hybrid energy system for locomotive application using particle swarm optimization," Journal of Energy Storage, vol. 19 , pp. $247-259,2018$.

[11] M. Marzband, E. Yousefnejad, A. Sumper, and J. L. DomínguezGarcía, "Real time experimental implementation of optimum energy management system in standalone microgrid by using multi-layer ant colony optimization," International Journal of Electrical Power and Energy Systems, vol. 75, pp. 265 - 274, 2016.

[12] M. Marzband, S. S. Ghazimirsaeid, H. Uppal, and T. Fernando, "A realtime evaluation of energy management systems for smart hybrid home microgrids," Electric Power Systems Research, vol. 143, pp. 624 - 633 , 2017.
[13] M. Elsied, A. Oukaour, T. Youssef, H. Gualous, and O. Mohammed, "An advanced real time energy management system for microgrids," Energy, vol. 114, pp. $742-752,2016$.

[14] S. A. Arefifar, M. Ordonez, and Y. A. I. Mohamed, "Energy management in multi-microgrid systems-development and assessment," IEEE Transactions on Power Systems, vol. 32, no. 2, pp. 910-922, 2017.

[15] S. Sikkabut, P. Mungporn, C. Ekkaravarodome, N. Bizon, P. Tricoli, B. Nahid-Mobarakeh, S. Pierfederici, B. Davat, and P. Thounthong, "Control of high-energy high-power densities storage devices by li-ion battery and supercapacitor for fuel cell/photovoltaic hybrid power plant for autonomous system applications," IEEE Transactions on Industry Applications, vol. 52, no. 5, pp. 4395-4407, 2016.

[16] A. Saez-de-Ibarra, V. I. Herrera, A. Milo, H. Gaztañaga, I. EtxeberriaOtadui, S. Bacha, and A. Padrós, "Management strategy for market participation of photovoltaic power plants including storage systems," IEEE Transactions on Industry Applications, vol. 52, no. 5, pp. 42924303, 2016.

[17] L. Han, T. Morstyn, and M. McCulloch, "Incentivizing prosumer coalitions with energy management using cooperative game theory," IEEE Transactions on Power Systems, vol. 34, no. 1, pp. 303-313, 2019.

[18] C. Olk, D. U. Sauer, and M. Merten, "Bidding strategy for a battery storage in the german secondary balancing power market," Journal of Energy Storage, vol. 21, pp. 787 - 800, 2019.

[19] A. Berrueta, A. Soto, M. García, Í. de la Parra, P. Sanchis, and A. Ursúa, "Critical comparison of energy management algorithms for lithiumion batteries in renewable power plants," in 2019 IEEE International Conference on Environment and Electrical Engineering and 2019 IEEE Industrial and Commercial Power Systems Europe (EEEIC / I CPS Europe), 2019, pp. 1-6.

[20] G. Lv, L. Wei, W. Li, S. Zhu, and J. Zheng, "Research on hybrid energy storage configuration in industrial estate," in 2017 20th International Conference on Electrical Machines and Systems (ICEMS), 2017, pp. $1-6$.

[21] K.-P. Kairies, D. Magnor, and D. U. Sauer, "Scientific measuring and evaluation program for photovoltaic battery systems (WMEP PVSpeicher)," Energy Procedia, vol. 73, pp. 200 - 207, 2015

[22] AEMET and Gobierno de Navarra. Meteorología y climatología de Navarra. Online. [Online]. Available: http://meteo.navarra.es/estaciones/ mapadeestaciones.cfm

[23] A. Berrueta, V. Irigaray, P. Sanchis, and A. Ursúa, "Lithium-ion battery model and experimental validation," in EPE'15 ECCE-Europe, 2015

[24] A. Berrueta, A. Urtasun, A. Ursúa, and P. Sanchis, "A comprehensive model for lithium-ion batteries: From the physical principles to an electrical model," Energy, vol. 144, pp. 286-300, 2018.

[25] J. Schmalstieg, S. Käbitz, M. Ecker, and D. U. Sauer, "A holistic aging model for li(nimnco)o $\mathrm{O}_{2}$ based 18650 lithium-ion batteries," Journal of Power Sources, vol. 257, pp. 325 - 334, 2014

[26] R. Bellman, Dynamic Programming, 1st ed. Princeton, NJ, USA Princeton University Press, 1957.

[27] A. Soto, A. Berrueta, P. Sanchis, and A. Ursúa, "Influence of renewable power fluctuations on the lifetime prediction of lithium-ion batteries in a microgrid environment," in 2019 IEEE International Conference on Environment and Electrical Engineering and 2019 IEEE Industrial and Commercial Power Systems Europe (EEEIC / I CPS Europe), 2019, pp. $1-6$. 